\title{
Modelo Hidrológico Distribuído para Previsão de Vazões Incrementais na Bacia do Rio Paranaíba entre Itumbiara e São Simão
}

\author{
Walter Collischonn, Carlos Eduardo Morelli Tucci, Robin Thomas Clarke, Martin Delgado Corbo \\ Benedito Cláudio da Silva, Bruno Collischonn, Daniel Allasia, Adriano Rolim da Paz \\ Instituto de Pesquisas Hidráulicas - UFRGS \\ collischonn@iph.ufrgs.br; tucci@iph.ufrgs.br
}

Recebido: 01/02/06 - revisado: 31/08/06 - aceito: 13/12/06

\begin{abstract}
RESUMO
Este artigo apresenta uma metodologia de previsão de vazão em tempo real baseada em um modelo hidrológico distribuído, utilizando informações de precipitação observada em postos pluviométricos e precipitação prevista pelo modelo meteorológico ETA em funcionamento no Centro de Previsão de Tempo e Estudos Climáticos do Instituto Nacional de Pesquisas Espaciais. Um esquema de atualização de variáveis do modelo hidrológico é aplicado utilizando dados de vazão que estariam disponíveis em uma aplicação operacional. A metodologia foi avaliada na bacia do rio Paranaíba, na região incremental entre as usinas hidrelétricas de Itumbiara e São Simão. Os resultados indicam que a inclusão de previsões quantitativas de chuva poderá ter um impacto significativo na redução dos erros da previsão de vazão realizada operacionalmente pelas entidades e empresas do setor elétrico nesta bacia.
\end{abstract}

Palavras-chave: modelo hidrológico distribuído, previsão de vazões, previsão de precipitação.

\section{INTRODUÇÃO}

A energia elétrica no Brasil é basicamente produzida em usinas hidrelétricas. Ao contrário da média mundial, em que a geração hídrica representa em torno de $20 \%$ do total da energia elétrica produzida, no Brasil este percentual atinge valores próximos de $90 \%$. As usinas hidrelétricas no Brasil estão ligadas aos grandes centros consumidores através de redes de transmissão, num sistema quase que completamente interligado em escala nacional, incluindo ainda as usinas termoelétricas. A configuração do Sistema Interligado Nacional (SIN) permite que uma determinada demanda de energia possa ser atendida de diferentes formas, por diferentes usinas de geração, sujeita a determinadas restrições de geração e transmissão.

As decisões sobre a operação de todos os reservatórios deste sistema são tomadas pelo Operador Nacional do Sistema Elétrico Interligado (ONS). Para a tomada de decisão são utilizadas ferramentas como modelos de otimização, cujo objetivo é reduzir o custo de produção de energia, por exemplo, minimizando a utilização de centrais termoelétricas, cujo custo de produção é tipicamente mais elevado do que o de centrais hidrelétricas.
Os modelos de otimização são fortemente dependentes de previsões sobre a demanda e a oferta (volume de água) de energia no futuro. Para definir a operação dos reservatórios do SIN são utilizadas previsões com horizonte de alguns dias até alguns anos (Livino de Carvalho et al., 2001; Pimentel et al., 2001; Maceira et al., 1997).

As previsões com horizonte inferior a um mês são especialmente importantes neste contexto, porque são utilizadas para definir a meta de geração hidrelétrica ou termelétrica de cada usina do sistema (Maceira et al., 1997). Previsões de vazão neste horizonte de tempo são geradas operacionalmente pelo ONS com base em modelos estocásticos do tipo vazão x vazão (Maceira et al., 1997; Damázio e Maceira, 1995). Este tipo de modelo tem a vantagem da simplicidade, e produz bons resultados quando a bacia possui memória longa, ou seja, quando o escoamento na bacia se dá de forma lenta. Assim, em bacias onde predomina o armazenamento subterrâneo ou a onde a declividade é baixa, as previsões podem ser consideradas satisfatórias. Por outro lado, em bacias com resposta mais rápida à chuva, como muitas bacias do Sul do Brasil, os erros destes modelos são consideráveis.

Para obter melhores previsões em bacias de resposta rápida, com memória curta, pode ser conveniente incluir no modelo de previsão as informa- 
ções de chuva observada ou prevista (Bertoni et al., 1992). A simples inclusão das informações de chuva observada pode melhorar as previsões de modelos estatísticos, como mostram os resultados de Guilhon (2002) na bacia do rio Iguaçu. A incorporação de previsões quantitativas de chuva na metodologia de previsão de vazão pode trazer benefícios econômicos através da diminuição de vertimentos desnecessários, da redução do risco de inundações e de uma melhor operação do sistema.

Alguns estudos mostram que a previsão de vazão em curto prazo pode melhorar quando se consideram as previsões meteorológicas de precipitação como entrada dos modelos hidrológicos, aumentando a informação e a antecipação da previsão (Yu et al., 1999; Yates et al., 2000; Damrath et al., 2000; Ibbitt et al., 2000, Andreolli, 2003; Collischonn et al., 2005; Tucci et al., 2003).

No entanto, a precipitação é, provavelmente, a variável mais difícil de se prever utilizando os modelos numéricos de previsão de tempo e clima, mas o desenvolvimento destes modelos e dos recursos computacionais vem permitindo avanços na previsão quantitativa de precipitação e de outras variáveis (Hollingsworth, 2003; Mao et al., 2000; McBride e Ebert, 2000; Collier e Krzysztofowicz, 2000; Damrath et al., 2000; Golding, 2000). Em função destes avanços já existem casos em que as previsões quantitativas de precipitação de modelos meteorológicos são utilizadas operacionalmente para previsões de vazão em tempo real, com resultados promissores (Bremicker et al., 2004).

Neste trabalho é apresentada uma metodologia de previsão de vazão baseada na previsão quantitativa de chuva, utilizando um modelo hidrológico distribuído para representar a transformação da chuva em vazão. São apresentados resultados obtidos em testes de previsão realizados para o rio Paranaíba, na bacia incremental entre Itumbiara e São Simão.

\section{METODOLOGIA DE PREVISÃO}

A metodologia de previsão de vazão utilizando a previsão de chuva empregada neste trabalho está baseada na utilização de um modelo hidrológico distribuído que representa os processos de geração de escoamento na bacia a partir da chuva observada e prevista, e de propagação deste escoamento ao longo da rede de drenagem da bacia. $\mathrm{O}$ modelo hidrológico utilizado é o MGB-IPH (Collischonn e Tucci, 2001), com algumas adaptações descritas brevemente a seguir.
Os parâmetros do modelo hidrológico são calibrados utilizando as chuvas observadas nos postos pluviométricos, procurando descrever da melhor forma possível os hidrogramas no exutório, bem como em postos fluviométricos intermediários e em afluentes.

$\mathrm{Na}$ aplicação do modelo para previsão são utilizadas as chuvas observadas e as chuvas previstas, como descrito em Andreolli (2003) e Tucci et al. (2003). Considerando que o tempo em que inicia a previsão é denominado $\mathrm{t}_{0}$, para cada ciclo de previsão de vazão o modelo hidrológico é iniciado num intervalo de tempo $\mathrm{t}_{0}-\mathrm{t}_{\mathrm{a}}$ e é executado até o tempo $\mathrm{t}_{0}$ com dados de chuva observados, sendo desta forma $\mathrm{t}_{\mathrm{a}}$ o tempo de "aquecimento" do modelo. No tempo $\mathrm{t}_{0} \mathrm{o}$ modelo começa a ser executado, recebendo como dado de entrada a previsão de chuva. O modelo gera resultados até o tempo $t_{\mathrm{h}}$ que é o tempo total de previsão, ou o horizonte de previsão. Esta situação procura representar, de forma realista, a situação de previsão operacional, em que os dados de chuva observada estão disponíveis para o passado e os dados de chuva prevista estão disponíveis para o futuro.

Durante o tempo de aquecimento do modelo também ocorre o procedimento de atualização de variáveis, em que as vazões calculadas pelo modelo hidrológico são comparadas às vazões observadas em diversos pontos na bacia, conforme descrito no item específico a seguir.

\section{O modelo MGB-IPH}

O modelo hidrológico MGB-IPH é um modelo distribuído desenvolvido para aplicações em grandes bacias, isto é, com áreas superiores a, aproximadamente, $10.000 \mathrm{~km}^{2}$.

O modelo subdivide a bacia em células regulares (geralmente quadradas), com tamanhos na ordem de $0,1 \times 0,1$ graus, o que equivale a, aproximadamente, $11 \times 11 \mathrm{~km}$. O tipo de vegetação e uso da terra dentro de cada célula é categorizada dentro de uma ou mais classes de acordo com a escolha do usuário. Para reduzir o custo computacional, foi utilizada a abordagem da Unidade de Resposta Agrupada (URA) (Grouped Response Unit - GRU) proposta por Kouwen et al. (1993). A URA consiste em agrupar todas as áreas com combinações similares de solo e uso da terra, de forma que a célula contenha um limitado número de diferentes URAs. Assim, o balanço de água é calculado para cada URA de cada célula, e as vazões estimadas em cada URA são posteriormente somadas e propagadas até a rede de drenagem. Esta abordagem vem sendo utilizada por vários modelos de grandes bacias, tais como VIC 
(Wood et al., 1992; Liang et al., 1994; Nijssem et al., 1997) e WATFLOOD (Soulis et al., 2004).

O balanço de água no solo é realizado utilizando uma metodologia baseada na geração de escoamento superficial por excesso de capacidade de armazenamento, porém com uma relação probabilística entre a umidade do solo e a fração de área de solo saturada. Esta relação tem sido usada em diversos modelos hidrológicos, especialmente em modelos de grandes bacias (Moore e Clarke, 1981; Zhao et al., 1992, Todini, 1996, Arnell, 1999, Bremicker, 1998; Lettenmaier, 2000).

A evapotranspiração é estimada, para cada URA em cada célula, através do método de Penman - Monteith (Shuttleworth, 1993), de forma semelhante ao modelo apresentado por Wigmosta et al. (1994). Valores dos parâmetros necessários para o cálculo da evapotranspiração, bem como detalhes sobre a influência da umidade do solo na evapotranspiração são apresentados em Collischonn e Tucci (2001). A resistência superficial (conjunto da resistência estomática do dossel) depende da disponibilidade de água no solo. Em condições favoráveis, os valores de resistência superficial são mínimos para uma dada cobertura vegetal. Nestas condições os valores de resistência superficial utilizados no modelo seguem estimativas disponíveis na literatura (Collischonn e Tucci, 2001). Para cultivos agrícolas admitiu-se um valor igual a $70 \mathrm{s.m}^{-1}$, que corresponde, aproximadamente, ao da grama, para cerrado foi adotado o valor de $90 \mathrm{s.m} \mathrm{m}^{-1}$ e para a água o valor de 0 s.m $\mathrm{m}^{-1}$.

Durante períodos de estiagem mais longos, a umidade do solo vai sendo retirada por transpiração e, à medida que o solo vai perdendo umidade, ocorre o stress hídrico, isto é, a transpiração diminui, mas a redução não ocorre imediatamente. Para valores de umidade do solo entre a capacidade de campo e um limite, que vai de 50 a $80 \%$ da capacidade de campo, a evapotranspiração não é afetada pela umidade do solo. A partir deste limite a evapotranspiração é diminuída, atingindo o mínimo normalmente zero - no ponto de murcha (Shuttleworth, 1993).

De acordo com o modelo proposto por Wigmosta et al. (1994), esta diminuição da evapotranspiração com a redução da umidade do solo ocorre pelo aumento da resistência superficial. A resistência superficial original é alterada por um coeficiente de ajuste $\left(\mathrm{F}_{4}\right)$. O coeficiente é igual a 1 , da saturação até um limite mínimo de armazenamento no solo (WL), a partir do qual seu valor começa a aumentar.

$r_{s u}=F_{4} \cdot r_{s}$ onde

$$
\frac{1}{F_{4}}=\frac{W-W_{P M}}{W_{L}-W_{P M}} \text { para } \quad \mathrm{W} \leq \mathrm{W}_{\mathrm{L}}
$$

onde $\mathrm{r}_{\mathrm{su}}$ [s.m-1] é a resistência superficial considerando a umidade do solo; $\mathrm{r}_{\mathrm{s}}$ [s.m-1] é a resistência superficial em boas condições de umidade do solo; $\mathrm{F}_{4}$ é um coeficiente de ajuste da resistência superficial; W [mm] é o armazenamento do solo; $\mathrm{W}_{\mathrm{PM}}$ [mm] é o armazenamento do solo no ponto de murcha e $W_{L}$ [mm] é o armazenamento em que inicia o efeito sobre a resistência superficial. Seguindo os valores típicos citados por Shuttleworth (1993), os parâmetros $\mathrm{W}_{\mathrm{L}}$ e $\mathrm{W}_{\mathrm{PM}}$ são fixados em $50 \%$ e $10 \%$ de $\mathrm{Wm}$, respectivamente, e não são considerados na calibração do modelo MGB-IPH.

A demanda de evapotranspiração é primeiramente atendida pela água interceptada considerando a resistência superficial nula, depois pela camada mais superficial do solo e, finalmente, se houver demanda adicional, é atendida pela camada mais profunda do solo. $\mathrm{O}$ valor do coeficiente $\mathrm{F}_{4}$ é calculado de forma independente para cada camada.

A propagação da vazão nos rios é realizada utilizando o método de Muskingun-Cunge linear (Tucci, 1998); já a propagação de vazão no interior de cada célula é realizada utilizando a metodologia do reservatório linear simples, em três processos paralelos: escoamento superficial, escoamento subsuperficial e escoamento subterrâneo.

O passo de tempo normalmente utilizado é de um dia, porque dificilmente estão disponíveis séries de dados para grandes bacias com intervalo de tempo menor, embora alguns processos de cálculo internos, como a propagação de ondas de cheia nos rios, utilizem intervalos de tempo de algumas horas.

A variabilidade espacial da precipitação é considerada através da interpolação dos dados dos postos pluviométricos para cada uma das células.

A variabilidade dos tipos de solos e do seu uso e cobertura vegetal é considerada através da utilização de planos de informação classificados a partir de imagens de satélite, ou mapas digitalizados. A variabilidade espacial do relevo é considerada através da utilização do modelo numérico do terreno (MNT) da região da bacia. O MNT utilizado tem, sempre, uma resolução espacial maior do que a resolução espacial das células do modelo. Desta forma há uma grande quantidade de informações do relevo no interior de cada célula, e esta informação é utilizada para regionalizar alguns parâmetros do modelo. 
Embora alguns processos sejam representados de forma conceitual, o modelo hidrológico conta com uma considerável base física, o que fortalece a relação entre os parâmetros e as características físicas da bacia. Durante este trabalho foi desenvolvida uma nova versão do modelo que conta com discretização do solo em duas camadas na vertical. Esta mudança foi necessária em função dos tipos de solos da região, que, em alguns casos, apresentam profundidades muito altas. A modificação realizada no modelo foi descrita e testada por Collischonn et al. (2006).

\section{Atualização das variáveis}

A simulação de um sistema hidrológico é afetada pelas incertezas nos dados de entrada, simplificações no modelo e técnicas inadequadas para a estimativa dos parâmetros, que, finalmente se refletem na estimativa dos parâmetros do modelo de simulação (Haan, 1989). Em conseqüência disso, uma previsão realizada utilizando como condição inicial as vazões calculadas pelo modelo tende a apresentar erros já nos primeiros intervalos de tempo, como pode ser observado na Figura 1a.

O objetivo da atualização das variáveis é modificar as condições iniciais do modelo, de forma a minimizar o erro no momento em que se inicia a previsão (instante $\mathrm{t}_{0}$ ). $\mathrm{O}$ efeito da atualização é mais importante nos primeiros intervalos de tempo da previsão (Figura 1b), mas em alguns casos pode se estender até algumas semanas.
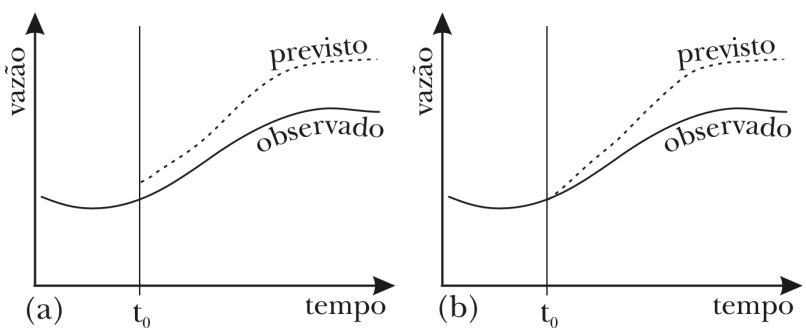

Figura 1 - Comparação entre previsão sem atualização (a) e com atualização (b).

A técnica de atualização implementada no modelo distribuído MGB-IPH foi desenvolvida a partir da técnica inicalmente apresentada por Andreolli et al. (2006) e Collischonn et al. (2005), e está baseada na utilização de dados de vazão em diferentes locais da bacia (disponíveis em tempo real nos postos telemétricos). Os valores de vazão calculados pelo modelo são comparados com os valores de vazão observados em cada um dos postos, gerando um Fator de Correção de Atualização
(FCA) conforme a Equação 2. A comparação é realizada tomando como base um período de $\mathrm{m}$ intervalos de tempo (dias) antes do início da previsão. Do tempo $t_{a}=t_{0}-m$ até o tempo $t_{0}$ são somadas as vazões observadas e as vazões calculadas. A relação entre os somatórios é o FCA, que é diferente para cada local em que existem dados fluviométricos.

$\mathrm{FCA}=\sum_{\mathrm{k}=\mathrm{t}_{0}-\mathrm{t}_{\mathrm{a}}}^{\mathrm{t}_{0}} \mathrm{Q}_{\mathrm{obs}}^{\mathrm{k}} / \sum_{\mathrm{k}=\mathrm{t}_{0}-\mathrm{t}_{\mathrm{a}}}^{\mathrm{t}_{0}} \mathrm{Q}_{\text {calc }}^{\mathrm{k}}$

onde $Q_{\text {obs }}$ é a vazão observada, $Q_{\text {calc }}$ é a vazão calculada; e o índice $\mathrm{k}$ indica o intervalo de tempo.

A ponderação ao longo de $\mathrm{m}$ intervalos de tempo para o cálculo do FCA é importante para evitar que erros aleatórios pontuais nas vazões medidas possam influenciar consideravelmente a correção dos valores calculados. $\mathrm{O}$ valor de $\mathrm{t}_{\mathrm{a}}$ pode ser definido de acordo com a confiabilidade dos dados individuais. Se a estimativa de vazão apresenta ruído, como é o caso quando a vazão é estimada a partir do balanço hídrico de reservatórios, o valor de $\mathrm{t}_{\mathrm{a}}$ deve ser relativamente alto ( 5 a 10 dias). Se a estimativa de vazão apresenta pouco ruído e é altamente confiável, o valor de $\mathrm{t}_{\mathrm{a}}$ pode ser de apenas 1 dia.

Em geral, os locais em que existem dados de vazão correspondem aos exutórios das sub-bacias, o que significa que pode ser calculado um fator FCA para cada sub-bacia. O fator FCA é aplicado, posteriormente, para corrigir as vazões calculadas em todas as células do modelo distribuído (vazões que estão na rede de drenagem) e para corrigir a quantidade de água nos aqüíferos da bacia, representada no modelo por reservatórios lineares simples.

\section{Atualização das vazões dos rios}

Inicialmente, a vazão dos rios calculada pelo modelo é corrigida, em todas as células a montante da célula correspondente ao posto fluviométrico, utilizando o fator de correção FCA, utilizando a Equação 3.

$Q_{\text {atual }}=F C A \cdot Q_{\text {calc }} \cdot\left(\frac{A_{i}}{A_{p}}\right)+Q_{\text {calc }} \cdot\left(1-\frac{A_{i}}{A_{p}}\right)$

onde $Q_{\text {atual }}$ é a vazão atualizada; $A_{\mathrm{i}}$ é a área a montante da célula considerada; $A_{P}$ é a área de drenagem no posto onde é observada a vazão $Q_{\text {obs }}$.

A Equação 3 é aplicada célula por célula, corrigindo a vazão que está ocorrendo no rio interno à célula. Admite-se que a informação introduzida pela vazão observada no posto fluviométrico é mais confiável nas células mais próximas do posto fluvio- 
métrico, onde a relação entre a área de drenagem da célula (Ai) e a área de drenagem do posto fluviométrico (Ap) é mais próxima de 1. Nas células mais próximas à cabeceira da sub-bacia, onde predomina o segundo termo da Equação 3, admite-se que a vazão observada no posto agrega pouca informação e que a vazão calculada é até mais confiável do que a vazão observada, uma vez que o ponto de observação está muito distante.

\section{Atualização dos volumes de água subterrânea}

No modelo MGB-IPH a água que chega à rede de drenagem pode ter um de três tipos de origem: superficial; sub-superficial e subterrânea. No escoamento interno da célula estes três tipos de escoamento estão separados, mas quando atingem a rede de drenagem eles são agregados. Entretanto, o modelo mantém uma estimativa da fração do escoamento oriundo de cada um dos três tipos ao longo de toda a rede de drenagem e de toda a propagação (Collischonn e Tucci, 2001). Por exemplo, num determinado intervalo de tempo, em uma célula qualquer com a vazão de $300 \mathrm{~m}^{3} \cdot \mathrm{s}^{-1}$ escoando pelo rio durante uma cheia, a parcela de água oriunda do escoamento subterrâneo é de $2 \% \quad\left(6 \mathrm{~m}^{3} \cdot \mathrm{s}^{-1}\right)$, a parcela de água oriunda do escoamento sub-superficial é de $8 \%\left(24 \mathrm{~m}^{3} \cdot \mathrm{s}^{-1}\right)$ e a parcela de água oriunda do escoamento superficial é de $90 \%\left(270 \mathrm{~m}^{3} \cdot \mathrm{s}^{-1}\right)$. Já numa situação de estiagem na mesma célula, com a vazão de apenas $5 \mathrm{~m}^{3} . \mathrm{s}^{-1}$, mais de $99 \%$ da vazão escoando na rede de drenagem da célula é oriunda dos reservatórios subterrâneos das células a montante. Este cálculo é realizado de forma contínua, e para cada valor de vazão ao longo da rede de drenagem estão associados três outros valores $\mathrm{PS}_{\mathrm{i}}, \mathrm{PI}_{\mathrm{i}}, \mathrm{e} \mathrm{PB}_{\mathrm{i}}$, que correspondem, respectivamente, às parcelas superficial, sub-superficial e subterrânea da vazão.

Nas aplicações do modelo para previsão de vazão, durante as estiagens, quando a maior parte da vazão é oriunda do escoamento subterrâneo $\left(\mathrm{PB}_{\mathrm{i}}>\right.$ $50 \%$ - por exemplo), também é realizada a atualização dos volumes armazenados nos reservatórios que representam os aqüíferos (reservatório subterrâneo de cada célula) o que melhora muito as previsões durante os períodos sem chuva, uma vez que a recessão dos hidrogramas tende a ser bem representada pelo modelo. Neste caso a atualização está baseada no mesmo fator de correção (FCA - Equação 2), e é aplicada sobre o volume armazenado no reservatório linear que representa o armazenamento subterrâneo de cada célula da sub-bacia.

$$
V B_{a}=F C A^{\text {ebas }} \cdot V B_{c} \cdot\left(P B_{i}\right)+V B_{c} \cdot\left(1-P B_{i}\right)
$$

Nesta equação $\mathrm{VB}_{\mathrm{a}}$ é o volume no reservatório subterrâneo atualizado; $\mathrm{VB}_{\mathrm{c}}$ é o volume calculado; $\mathrm{PB}_{\mathrm{i}}$ é a parcela da vazão na célula i que é oriunda do escoamento subterrâneo; e ebas é um expoente com valor entre 0 e 1 , que determina se a correção vai ser rápida (valores próximos a 1) ou lenta (valores próximos de 0). Após alguns testes com valores do expoente ebas entre 0 e 1 foi adotado o valor de 0,2. Este valor corrige a vazão de forma estável e suficientemente rápida para as previsões realizadas com freqüência semanal.

Equações semelhantes à Equação 4 podem ser aplicadas para os reservatórios sub-superficial e superficial quando o intervalo de tempo de cálculo e o intervalo de tempo dos dados de vazão é de uma hora, ou menos (Collischonn et al., 2005). Para intervalos de tempo grandes, como 1 dia, a atualização dos volumes armazenados nos reservatórios superficial e sub-superficial pode gerar ruído, porque a atualização tende a amplificar pequenas perturbações aleatórias nos valores observados, o que pode diminuir a qualidade da previsão.

Também é importante destacar que o efeito da atualização é maior nos primeiros intervalos de tempo da previsão. No caso da bacia incremental entre Itumbiara e São Simão, após um a três dias a vazão dos rios percorre toda a rede de drenagem e não haveria benefício na atualização, exceto no caso das situações de vazões baixas, quando a correção dos volumes armazenados nos reservatórios subterrâneos permitiria boas previsões de estiagem por um período de mais de uma semana.

\section{APLICAÇÃO}

\section{Bacia do rio Paranaíba}

O rio Paranaíba é um dos principais formadores do rio Paraná. O Rio nasce no Estado de Minas Gerais a uma altitude de $1.140 \mathrm{~m}$, percorre uma extensão de $1.120 \mathrm{~km}$, até sua desembocadura no rio Paraná. Sua bacia hidrográfica abrange uma área de $220.195 \mathrm{~km}^{2}$, até a confluência com o rio Grande, onde é formado o rio Paraná. A maior parte da bacia hidrográfica está localizada no Estado de Goiás e no Distrito Federal (68\%) e o restante (32\%) em Minas Gerais. Seu percurso está definido em 3 trechos distintos: Alto Paranaíba, correspondente ao trecho da nascente até o km 370; Médio Paranaíba, do km 370 até a barragem de Cachoeira Dourada, com 370 km; Baixo Paranaíba, da barragem de Cachoeira Dourada até a sua foz (extensão de $380 \mathrm{~km}$ ). 
A bacia de estudo está localizada entre as usinas hidrelétricas de Itumbiara e São Simão, e totaliza uma área de drenagem de $76.746 \mathrm{~km}^{2}$. Os principais afluentes são os rios Prata e Tijuco pela margem esquerda, e os rios dos Bois, Preto e Meia Ponte, na margem direita. O Rio dos Bois recebe as contribuições dos rios Turvo e Verde.

Os solos na bacia são formados basicamente por Latossolos vermelho-escuros em relevo suave ondulado e outros solos desta classe em relevo plano. Ocorrem secundariamente, Podzólicos vermelho-amarelos, Latossolos roxos e Cambissolos. Os Latossolos são, em geral, profundos e, dependendo da topografia e das características geológicas da região, podem gerar relativamente pouco escoamento superficial e elevado escoamento de base. A vegetação original era composta, predominantemente, por Floresta Tropical (semi-caducifólia) e Cerrado, com áreas de transição ou tensão ecológica entre os dois tipos. Atualmente predominam as áreas de pastagem e agricultura.

A bacia do rio Paranaíba está localizada em uma região de altitudes que variam entre 1000 e 300 metros, sendo que as maiores altitudes são encontradas na região norte da bacia nas cabeceiras do rio Meia Ponte, localizadas no Distrito Federal. O regime hidrológico dos afluentes do Paranaíba, no trecho de estudo, apresenta sazonalidade bem definida. O período úmido está compreendido entre novembro e abril, com vazões médias máximas entre fevereiro e março. Os valores mínimos ocorrem entre agosto e outubro.

O tempo de concentração estimado para as bacias deste trecho é menor do que 2 dias, e considerando o tempo de propagação da onda de cheia pelo rio Paranaíba até a usina de São Simão, o tempo total de concentração estimado é de 2 a 3 dias.

\section{Discretização da bacia}

Os dados utilizados na discretização da bacia para a aplicação do modelo hidrológico foram: mapas topográficos, mapas de cobertura vegetal e uso do solo e mapas de tipos de solos. Os dados de topografia utilizados foram obtidos do modelo numérico do terreno do mundo inteiro disponibilizado pela NASA e USGS (http://srtm.usgs.gov/), obtido através de imagens de radar estereoscópicas da Terra.

O modelo numérico de alta resolução (90 m) foi reamostrado para a resolução de $100 \mathrm{~m}$ $\left(0,001^{\circ}\right)$ e em seguida determinadas as direções de fluxo correspondentes. Através de um procedimento de upscaling de direções de fluxo, foi determinada a direção de escoamento para cada célula e a rede de drenagem do modelo hidrológico. Empregou-se adaptação do algoritmo de Reed (2003) desenvolvida por Paz et al. (2006). Os comprimentos destes trechos de rio e suas declividades foram obtidos de cartas em escala 1:250.000.

Informações sobre o uso do solo e a cobertura vegetal foram obtidas a partir da classificação de imagens do satélite Landsat (sensor ETM7). Ao todo foram classificadas 8 cenas (uma cena cobre uma região de $180 \times 180 \mathrm{~km})$. As imagens foram classificadas em 8 classes (água, floresta, agricultura irrigada, agricultura, pastagem, cerrado, campo sujo e urbano). Posteriormente as classes agricultura, agricultura irrigada, campo sujo e pastagem foram fundidas em uma única classe que representa áreas de uso para a agropecuária. As classes cerrado e floresta também foram unidas em função da pequena fração da área da bacia coberta por estes tipos de vegetação. Os tipos de solos foram obtidos a partir da digitalização do mapa de solos do RADAM Brasil, carta Goiânia.

Do ponto de vista da hidrologia, especialmente no caso de previsão de vazões, a característica mais importante do solo é o potencial de geração de escoamento superficial. Neste sentido, destacam-se os solos litólicos, que são geralmente rasos e tendem a gerar escoamento superficial mesmo para chuvas menos intensas. Os solos Glei também podem gerar escoamento superficial de forma intensa, porque ocorrem em áreas em que o lençol freático está relativamente próximo da superfície. Os cambissolos e os solos podzólicos encontram-se numa faixa intermediária de potencial de geração de escoamento superficial e, finalmente, os latossolos e as areias quartzosas são definidos como solos de baixo potencial de geração de escoamento superficial.

Assim, os solos da bacia foram classificados em três grupos: baixo, médio e alto potencial de escoamento superficial. No grupo de solos de alto potencial foram incluídos os solos litólicos e os solos Glei; no grupo de médio potencial foram incluídos os solos podzólicos e os cambissolos; no grupo de solos de baixo potencial foram incluídos os latossolos e as areias quartzosas. Esta redução do número de grupos de solos é necessária para evitar um número excessivo de URAs, que são resultantes da combinação dos solos com as classes de vegetação e uso do solo.

As URAs foram definidas a partir da combinação do mapa de cobertura vegetal e uso do solo e do mapa de tipos de solos. Esta combinação resultou em um grande número de classes que foram reagrupadas conforme a Tabela 1, que apresenta também a porcentagem de cada bloco na bacia. 
Tabela 1 - URAs ou blocos de comportamento hidrológico na bacia do rio Paranaíba.

\begin{tabular}{|l|l|}
\hline Classe de uso e cobertura & $\begin{array}{l}\text { Fração da área } \\
\text { da bacia (\%) }\end{array}$ \\
\hline Cerrado - Médio/Alto Potencial & 5,7 \\
\hline Cerrado - Baixo Potencial & 9,2 \\
\hline Agricultura - Baixo Potencial & 59,3 \\
\hline Agricultura - Médio Potencial & 20,1 \\
\hline Agricultura - Alto Potencial & 4,4 \\
\hline Água & 1,3 \\
\hline
\end{tabular}

\section{Dados hidrológicos utilizados}

As séries de precipitação da área de estudo foram disponibilizadas pelo ONS, totalizando dados de 26 postos de medição, incluindo estações telemétricas e convencionais. Esta é a rede de pluviômetros que, de acordo com o ONS, poderia ser adaptada para operar em tempo real em um futuro relativamente próximo.

Os dados de vazão utilizados no trabalho incluem dados de postos fluviométricos, e dados de vazão incremental natural. As séries de vazões naturais correspondem a dados que não são medidos diretamente, mas são reconstituídos a partir dos dados observados nos reservatórios, das informações sobre a operação de reservatórios e sobre as retiradas de água para usos consuntivos. As séries de vazão natural tentam representar os dados de vazão que seriam observados nos locais dos barramentos, se não existissem as interferências antrópicas. Estas vazões são utilizadas pelo Operador Nacional do Sistema Hidrelétrico (ONS) para realizar previsões de vazão nos reservatórios das usinas que compõem o SIN e, com o auxilio destas previsões, planeja-se a operação de todo o sistema.

A vazão média do rio Paranaíba em São Simão no período de 1960 a 2001, estimada a partir dos dados de vazão natural fornecidos pelo ONS, é de $2495 \mathrm{~m}^{3} \cdot \mathrm{s}^{-1}$. A vazão média incremental no trecho entre Itumbiara e São Simão é de $960 \mathrm{~m}^{3} \cdot \mathrm{s}^{-1}$, no mesmo período, o que significa que $39 \%$ da vazão que chega a São Simão é gerada no trecho a jusante de Itumbiara. Em 1982 ocorreu a máxima vazão incremental da série, superando $6200 \mathrm{~m}^{3} \cdot \mathrm{s}^{-1}$, em um evento de cheia em que a máxima vazão natural em São Simão foi de mais de $13.000 \mathrm{~m}^{3} \cdot \mathrm{s}^{-1}$, o que mostra que a região analisada neste trabalho é fundamental na formação das grandes cheias.

O modelo MGB-IPH realiza o cálculo da evaporação e da evapotranspiração com base em dados meteorológicos observados. As variáveis utilizadas são temperatura, pressão atmosférica, umida- de relativa do ar, radiação solar ou insolação e velocidade do vento. Foram identificadas 7 estações meteorológicas operadas pelo INMET nesta bacia e os dados médios mensais (normais climatológicas) de cada um destes postos foram utilizados nas simulações e nas previsões com o modelo.

Os dados de precipitação prevista pelo modelo regional ETA do CPTEC foram disponibilizados com uma resolução espacial de 0,4 x 0,4 graus, em intervalo de tempo diário, e com freqüência semanal. Cada uma das previsões cobre um período de 10 dias que se estende da quarta-feira de uma semana (inclusive) até a sexta-feira feira da semana seguinte (inclusive). O período de análise de 1996 a 2001 tem 313 semanas.

\section{CALIBRAÇ̃̃O DO MODELO}

Antes da utilização para previsão de vazão, o modelo hidrológico foi calibrado utilizando os dados de chuva observada nos postos pluviométricos. A calibração do modelo hidrológico é realizada em três fases: 1) estimativa inicial dos parâmetros a partir de bacias já calibradas com características físicas semelhantes; 2) calibração manual; 3) calibração automática. Estas fases são descritas nos parágrafos a seguir.

A estimativa inicial dos parâmetros é realizada considerando que os parâmetros devem ter valores próximos daqueles encontrados em aplicações anteriores do mesmo modelo em bacias de características semelhantes. Esta estimativa inicial pode ter resultados muito bons quando as duas bacias estão localizadas em uma região bastante homogênea do ponto de vista da geologia, do clima, do relevo e da pedologia (Collischonn e Tucci, 2003; Allasia et al., 2006).

A partir da estimativa inicial, é iniciada uma fase de calibração manual, baseada principalmente na análise dos hidrogramas calculados e observados. Quando uma representação razoavelmente boa dos hidrogramas é atingida, inicia-se a terceira fase da calibração, que é a calibração automática. A calibração automática é realizada utilizando o algoritmo evolutivo multi-objetivo MOCOM-UA, desenvolvido por Yapo et al. (1998), descrito também em Collischonn e Tucci (2003).

As funções-objetivo normalmente consideradas na calibração do modelo MGB-IPH com o algoritmo MOCOM-UA são o coeficiente de eficiência de Nash Sutcliffe (Nash e Sutcliffe, 1970) (RNS), o coeficiente de Nash Sutcliffe dos logaritmos das vazões RNSlog e o erro percentual de volumes $(\Delta \mathrm{V})$ : 
Modelo Hidrológico Distribuído para Previsão de Vazões Incrementais na Bacia do Rio Paranaíba entre Itumbiara e São Simão

$$
\begin{aligned}
& \mathrm{RNS}=1-\frac{\sum_{\mathrm{t}=1}^{\mathrm{nt}}\left(\mathrm{Q}_{\mathrm{obs}}(\mathrm{t})-\mathrm{Q}_{\mathrm{cal}}(\mathrm{t})\right)^{2}}{\sum_{\mathrm{t}=1}^{\mathrm{nt}}\left(\mathrm{Q}_{\mathrm{obs}}(\mathrm{t})-\overline{\mathrm{Q}_{\mathrm{obs}}}\right)^{2}} \\
& \mathrm{RNS}_{\log }=1-\frac{\sum_{\mathrm{t}=1}^{\mathrm{nt}}\left(\log \left(\mathrm{Q}_{\mathrm{obs}}(\mathrm{t})\right)-\log \left(\mathrm{Q}_{\mathrm{cal}}(\mathrm{t})\right)\right)^{2}}{\sum_{\mathrm{t}=1}^{\mathrm{nt}}\left(\log \left(\mathrm{Q}_{\mathrm{obs}}(\mathrm{t})\right)-\overline{\left.\log \left(\mathrm{Q}_{\mathrm{obs}}\right)\right)^{2}}\right.} \\
& \Delta \mathrm{V}=\frac{\sum_{\mathrm{t}=1}^{\mathrm{nt}}\left(\mathrm{Q}_{\mathrm{cal}}(\mathrm{t})-\mathrm{Q}_{\mathrm{obs}}(\mathrm{t})\right)}{\sum_{\mathrm{t}=1}^{\mathrm{nt}}\left(\mathrm{Q}_{\text {obs }}(\mathrm{t})\right)}
\end{aligned}
$$

onde t é o intervalo de tempo; nt é o número de intervalos de tempo; $\mathrm{V}$ é o volume $\left(\mathrm{m}^{3}\right) ; \Delta \mathrm{V}$ é o erro relativo adimensional deste volume; $Q_{\text {cal }}$ é a vazão calculada no posto; $Q_{\mathrm{obs}}$ é a vazão observada; e $\overline{Q_{\text {obs }}}$ é a média das vazões observadas.

O período de dados utilizado na calibração foi de 1991 a 2001, e a calibração foi realizada de forma independente para cada sub-bacia. A verificação foi realizada com os dados do período de 1980 a 1990. Os resultados foram avaliados nos postos fluviométricos utilizados, considerando as funçõesobjetivo dadas pelas Equações 5 a 7 . Os resultados estão na Tabela 2. A Figura 2 apresenta os hidrogramas calculado e observado em São Simão (apenas vazão incremental) no ano de 1982, durante o período de verificação.

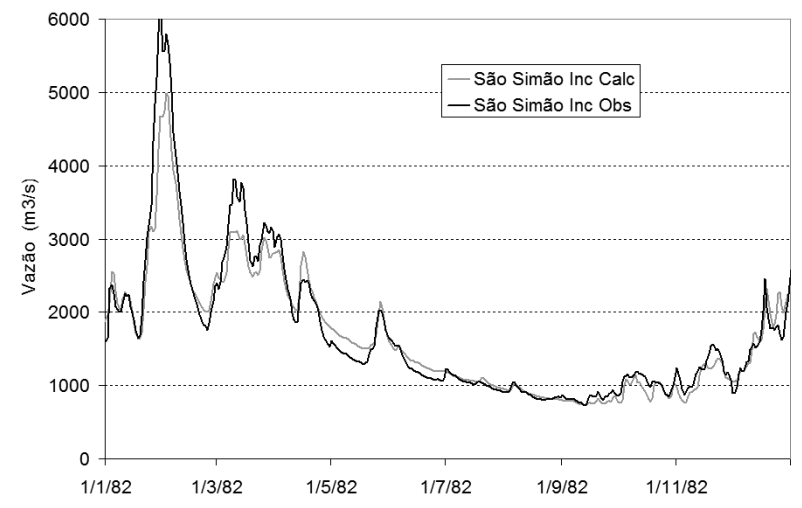

Figura 2 - Hidrogramas de vazão incremental a São Simão calculada e observada no período de verificação.

Observa-se que os hidrogramas calculados ajustam-se relativamente bem aos observados. Há uma pequena tendência de subestimar os picos no ano de 1982, mas não há uma queda expressiva de desempenho entre os períodos de calibração e de verificação. O maior pico de vazão do período, ocorrido no período de verificação, em 1982, foi subestimado em cerca de $1000 \mathrm{~m}^{3} \cdot \mathrm{s}^{-1}$, o que pode ser considerado um erro significativo, uma vez que representa cerca de $20 \%$ da vazão de pico. Entretanto o tempo de subida e de descida do hidrograma são muito bem representados, e outros picos de cheia menores são muito bem ajustados. É provável que um aumento no número de postos pluviométricos na bacia permita reduzir os erros das simulações. Assim, com base nas estatísticas calculadas, como o coeficiente de Nash-Sutcliffe e os erros de volume, e com base na análise dos gráficos dos hidrogramas, a calibração pode ser considerada satisfatória.

\begin{tabular}{|c|c|c|c|c|c|c|c|c|c|}
\hline \multirow{2}{*}{ Rio } & \multirow{2}{*}{ Local } & \multirow{2}{*}{ Código } & \multirow{2}{*}{$\begin{array}{l}\text { Área } \\
\left(\mathrm{km}^{2}\right)\end{array}$} & \multicolumn{3}{|c|}{ Calibr. (1991-2001) } & \multicolumn{3}{|c|}{ Verific. (1981-1990) } \\
\hline & & & & NS & RNSlog & $\Delta \mathrm{V}$ & NS & NSlog & $\Delta \mathrm{V}$ \\
\hline Meia Ponte & Ponte Meia Ponte & 60680000 & 11.483 & 0,80 & 0,84 & 4,8 & 0,83 & 0,88 & $-10,7$ \\
\hline Meia Ponte & Ponte Go-206 & 60680004 & 12.256 & 0,79 & 0,84 & $-1,5$ & - & - & - \\
\hline Dos Bois & Fazenda Boa Vista & 60715000 & 4.569 & 0,62 & 0,69 & 13,8 & 0,68 & 0,82 & 0,0 \\
\hline Turvo & Faz. Nova do Turvo & 60750000 & 2.436 & 0,63 & 0,74 & 8,1 & 0,68 & 0,78 & $-16,4$ \\
\hline Verde & Ponte Rio Verdão & 60790000 & 8.651 & 0,80 & 0,85 & 6,2 & 0,80 & 0,81 & $-6,7$ \\
\hline Dos Bois & Abaixo B. R. Verde & 60805100 & 30.491 & 0,87 & 0,90 & 1,8 & 0,91 & 0,94 & $-6,3$ \\
\hline Preto & Quirinópolis & 60870000 & 1.657 & 0,58 & 0,53 & $-5,7$ & 0,44 & 0,45 & 10,6 \\
\hline Prata & Ponte do Prata & 60855000 & 5.266 & 0,72 & 0,84 & 1,3 & 0,76 & 0,82 & 0,3 \\
\hline Tijuco & Ituiutaba & 60845000 & 6.383 & 0,74 & 0,78 & $-10,0$ & 0,78 & 0,79 & 7,1 \\
\hline Tijuco & Cach. do Gambá & 60845500 & 6.998 & 0,64 & 0,68 & $-9,0$ & 0,80 & 0,84 & 1,0 \\
\hline Paranaíba & São Simão (increm.) & - & 76.746 & 0,90 & 0,89 & 0,6 & 0,92 & 0,90 & $-1,9$ \\
\hline
\end{tabular}

Tabela 2 - Valores das funções-objetivo na calibração e verificação do modelo (no posto GO 206 do rio Meia Ponte não existem dados observados de vazão no período de verificação). 

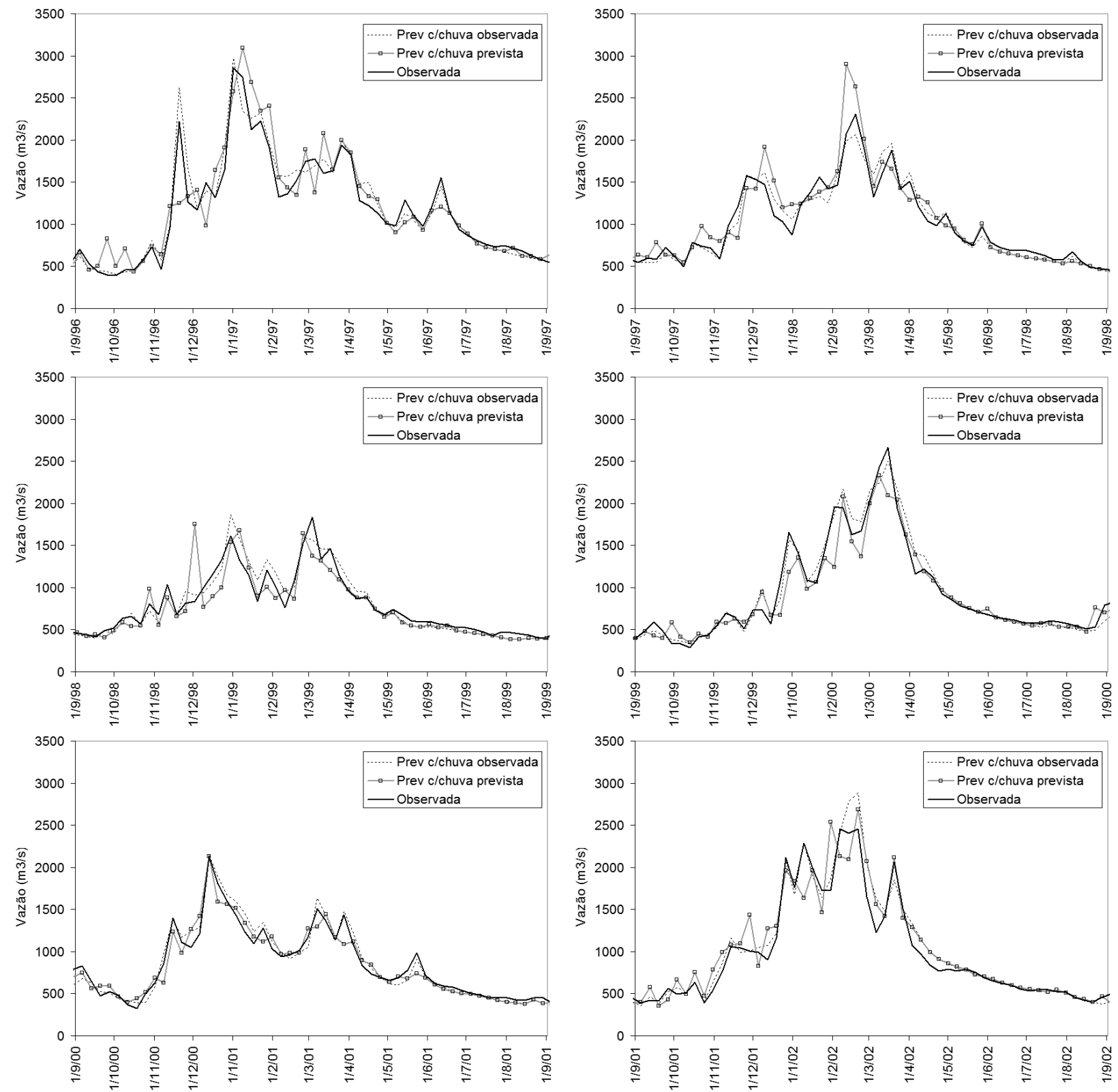

Figura 3 - Vazões incrementais médias semanais previstas e observadas na bacia incremental de São Simão (setembro 1996 a setembro 2002).

\section{RESULTADOS}

Após a calibração utilizando dados de chuva observada, o modelo hidrológico foi aplicado utilizando as informações de chuva observada e chuva prevista. O modelo foi aplicado utilizando chuvas observadas até a terça-feira (inclusive) de cada semana. A partir daí foram utilizadas as chuvas previstas pelo modelo ETA, e o modelo foi executado em cada semana por um período de 12 dias, isto é, da quarta-feira de uma semana até o domingo da semana seguinte, em intervalo de tempo diário. Nos dois últimos dias do horizonte de previsão a precipitação foi considerada nula.

A comparação mais adequada de um modelo de previsão seria a comparação com os resultados de um modelo alternativo, no caso o modelo atualmente utilizado para o planejamento do setor elétrico pelo ONS. Entretanto, estes dados não estavam disponíveis no momento da elaboração deste artigo. As previsões foram analisadas em intervalo de tempo 
diário e em intervalo semanal. São apresentados alguns dos resultados em intervalo semanal, considerando as estatísticas normalmente utilizadas no setor elétrico, e alguns resultados em intervalo de tempo diário, utilizando o coeficiente de NashSutcliffe.

As vazões médias semanais previstas e observadas foram calculadas para cada intervalo de sete dias que vai do sábado de uma semana até a sextafeira da semana seguinte. Os resultados das previsões foram analisados separadamente nos períodos de 1996 a 2001 e de 2002 a 2003. O período de 1996 a 2001 foi utilizado no desenvolvimento do modelo, e o período de 2002 a 2003 foi utilizado em testes posteriores, procurando reproduzir, da melhor forma possível, a situação operacional.

A Figura 3 apresenta a comparação das vazões médias semanais observadas e previstas ao longo do tempo, para os anos de 1996 a 2002. Observase que as previsões são boas durante o período de recessão (meses de julho a setembro). Nos períodos chuvosos ocorrem erros de subestimativa, como em novembro de 1996, e de superestimativa, como no início de dezembro de 1998. Porém, de maneira geral, as previsões indicam de forma adequada as grandes alterações ascendentes e descendentes da vazão.

As previsões obtidas utilizando a chuva observada também são apresentadas na Figura 3, o que permite comparar os resultados com as previsões obtidas a partir das chuvas previstas pelo modelo ETA. Assim é possível identificar falhas da previsão de chuva, como em novembro de 1996, quando o modelo baseado nas chuvas previstas pelo ETA não consegue prever o aumento da vazão, e como em fevereiro de 1998, quando a previsão superestima a vazão média da semana.

A Tabela 3 apresenta um resumo dos índices estatísticos utilizados para avaliar as previsões de vazão média semanal incremental a São Simão nos períodos de desenvolvimento do modelo (de 1996 a 2001) e de testes (2002 e 2003). No período de 1996 a 2001, o erro absoluto médio das previsões de vazão com base nas previsões de chuva do modelo ETA foi de $122 \mathrm{~m}^{3} . \mathrm{s}^{-1}$. O erro relativo médio foi de $12,7 \%$. No período de testes estes erros foram reduzidos para $113 \mathrm{~m}^{3} \cdot \mathrm{s}^{-1}$ e para $10,8 \%$, indicando que a qualidade das previsões foi mantida.

As previsões diárias foram avaliadas no período completo de 1996 a 2003, utilizando o coeficiente de Nash-Sutcliffe (Equação 5), considerando o tempo de antecedência da previsão. A Tabela 4 apresenta os resultados, considerando previsões obtidas a partir das chuvas previstas e das chuvas observadas, e para as antecedências de 1 a 12 dias. Para tornar mais claro o papel da atualização das variáveis do modelo hidrológico, foram realizadas previsões de vazão sem a atualização, e os resultados também são apresentados na tabela 4.

Tabela 3 - Índices estatísticos das previsões de vazão media semanal incremental a São Simão com base na chuva prevista pelo modelo ETA.

\begin{tabular}{|l|l|l|}
\hline Índice & $\begin{array}{l}1996- \\
2001\end{array}$ & $\begin{array}{l}2002- \\
2003\end{array}$ \\
\hline Erro médio $\left(\mathrm{m}^{3} \cdot \mathrm{s}^{-1}\right)$ & $-2,2$ & 15,6 \\
\hline Erro absoluto médio $\left(\mathrm{m}^{3} \cdot \mathrm{s}^{-1}\right)$ & 122 & 113 \\
\hline Erro relativo médio $\%)$ & 12,7 & 10,8 \\
\hline Coeficiente de correlação & 0,927 & 0,947 \\
\hline
\end{tabular}

De acordo com o esperado, a qualidade da previsão decai com o aumento da antecedência. Os valores do coeficiente de Nash-Sutcliffe são mais altos nos primeiros dias, com valores próximos a 0,96 , que superam, inclusive, o valor deste coeficiente obtido durante a calibração. Além disso, a Tabela 4 também mostra que a qualidade da previsão baseada na chuva prevista decai mais rapidamente do que a qualidade da previsão baseada na chuva observada. Isto está relacionado aos erros da previsão de chuva, e indica que ainda há espaço para aprimoramentos das previsões de chuva.

O efeito da atualização de variáveis sobre a qualidade das previsões pode ser observado comparando as duas últimas colunas da tabela 4 . $\mathrm{O}$ valor do coeficiente de Nash-Sutcliffe para o primeiro dia de previsão, no caso da previsão sem atualização, corresponde, aproximadamente, ao valor encontrado durante à calibração e verificação do modelo. As previsões realizadas com atualização apresentam um desempenho melhor nos primeiros dias, mas vão decaindo aos poucos, praticamente igualando-se às previsões sem atualização nos últimos dias do horizonte total de previsão.

\section{CONCLUSÕES E RECOMENDACÕOES}

Este artigo apresenta os resultados de um modelo de previsão de vazões na bacia incremental do rio Paranaíba entre Itumbiara e São Simão. Para a calibração do modelo foram utilizados dados de vazão em postos fluviométricos, uso do solo, relevo e tipos de solos da bacia. O modelo hidrológico foi calibrado e verificado utilizando dados de chuva observada, mostrando bons resultados tanto na va- 
Tabela 4 - Valores do coeficiente de Nash-Sutcliffe obtidos nas previsões de vazão utilizando previsões de chuva do modelo ETA e chuvas observadas nos postos pluviométricos, para antecedências de 1 a 12 dias, durante o período de 1996 a 2003.

\begin{tabular}{|l|l|l|l|}
\hline Antecedência & $\begin{array}{l}\text { Chuva observada } \\
\text { com atualização }\end{array}$ & $\begin{array}{l}\text { Chuva prevista } \\
\text { com atualização }\end{array}$ & $\begin{array}{l}\text { Chuva prevista sem } \\
\text { atualização }\end{array}$ \\
\hline 1 dia & 0,97 & 0,96 & 0,92 \\
\hline 2 dias & 0,96 & 0,94 & 0,90 \\
\hline 3 dias & 0,93 & 0,91 & 0,88 \\
\hline 4 dias & 0,93 & 0,88 & 0,86 \\
\hline 5 dias & 0,93 & 0,85 & 0,83 \\
\hline 6 dias & 0,93 & 0,84 & 0,81 \\
\hline 7 dias & 0,93 & 0,80 & 0,79 \\
\hline 8 dias & 0,94 & 0,78 & 0,76 \\
\hline 9 dias & 0,94 & 0,78 & 0,75 \\
\hline 10 dias & 0,91 & 0,75 & 0,73 \\
\hline 11 dias & 0,92 & 0,73 & 0,71 \\
\hline 12 dias & 0,92 & 0,72 & 0,70 \\
\hline
\end{tabular}

zão incremental total como na vazão observada em alguns dos principais afluentes do rio Paranaíba no trecho. A aplicação do modelo hidrológico para previsão utilizando chuvas previstas pelo modelo ETA em um período de testes resultou em um erro médio absoluto de $113 \mathrm{~m}^{3} . \mathrm{s}^{-1}$, e em um erro relativo médio de $10,8 \%$.

Algumas características importantes do modelo de previsão proposto são: 1) estabilidade dos resultados entre o período de calibração e verificação; 2) bons resultados também em bacias intermediárias; 3) não usa artifícios de correção das chuvas previstas, o que significa que melhores previsões de chuva vão gerar melhores previsões de vazão, sem exigir novas calibrações ou alterações da estrutura do modelo; 4) pode utilizar outras fontes de dados de previsão de chuva (outros modelos, versões do modelo ETA com mais alta resolução, etc).

A principal desvantagem do modelo é que ele é relativamente difícil de ser implementado e exige uma significativa quantidade de informações.

O impacto da introdução da técnica de atualização das variáveis no modelo sobre a qualidade das previsões não pode ser avaliado isoladamente neste texto e poderá ser explorado em um próximo trabalho.

\section{AGRADECIMENTOS}

Os autores agradecem as sugestões recebidas dos técnicos do ONS durante o desenvolvimento do trabalho que deu origem a este texto e aos revi- sores anônimos, que contribuíram com valiosos comentários para a elaboração da sua versão final.

\section{REFERÊNCIAS}

ALLASIA, D.; COLLISCHONN, W.; SILVA, B.C.; TUCCI, C.E.M., 2006. Large basin simulation experience in South America. IAHS Publ. n. 303, v. 303, p. 360370.

ANDREOLLI, I., 2003. Previsão de vazão em tempo real no rio Uruguai com base na previsão meteorológica. Dissertação de mestrado. IPH UFRGS. Porto Alegre.

ARNELL, N.W., 1999. A simple water balance model forthe simulation of streamflow over a large geographic domain. J. of Hydrol., v.217, p.314-335.

BERTONI, J.C.; TUCCI, C.E.M.; CLARKE, R.T., 1992. Rainfall-based real-time flood forecasting. J. of Hydrol., v.131, 313-339.

BREMICKER, M., 1998. Aufbau eines Wasserhaushaltsmodells für das Weser und das Ostsee Einzugsgebiet als Baustein eines Atmosphären-Hydrologie-Modells. Dissertation Doktorgrad, Geowissenschaftlicher Fakultät der Albert-Ludwigs-Universität. Freiburg.

BREMICKER, M.; HOMAGK, P.; LUDWIG, K., 2004. Operational low-flow-forecast for the Neckar river basin. Wasserwirtschaft 7-8, p. 40-46.

COLLIER, C.G.; KRZYSZTOFOWICZ, R., 2000. Quantitative precipitation forecasting. J. of Hydrol., v. 239, p. 1-2.

COLLISCHONN, W.; TUCCI, C..; CORBO, M.; SILVA, B., COLLISCHONN, B.; ALLASIA, D.; BRAVO, J., 2006. Modelo hidrológico de grandes bacias com duas 
Modelo Hidrológico Distribuído para Previsão de Vazões Incrementais na Bacia do

Rio Paranaíba entre Itumbiara e São Simão

camadas de solo. Submetido ao $20^{\circ}$ Congreso $\mathrm{Na}$ cional del Agua, Tucumán (Argentina).

COLLISCHONN, W.; HAAS, R.; ANDREOLLI, I.; TUCCI, C.E.M., 2005. Forecasting river Uruguay flow using rainfall forecasts from a regional weather-prediction model. J. of Hydrol., v. 305, p. 87-98.

COLLISCHONN, W.; TUCCI, C.E.M., 2003. Ajuste multiobjetivo dos parâmetros de um modelo hidrológico. Rev. Bras. de Rec. Hídricos, v.8, n.3.

COLLISCHONN, W.; TUCCl, C.E.M., 2001. Simulação hidrológica de grandes bacias. Rev. Bras. de Rec. Hídricos, v. 6, n. 1.

DAMÁZIO, J.M.; MACEIRA, M.E., 1995. Modelos de previsão hidrológica para uso na operação de sistemas hidrotérmicos de geração de energia elétrica. XI Simp. Bras. de Rec. Hidricos, ABRH, Recife.

DAMRATH, U.; DOMS, G.; FRÜHWALD, D.; HEISE, E.; RICHTER, B.; STEPPELER, J., 2000. Operational quantitative precipitation forecasting at the German Weather Service. J. of Hydrol., v. 239, p. 260-285.

GOLDING, B.W., 2000. Quantitative precipitation forecasting in the UK. J. of Hydrol., v. 239, p. 286-305.

GUILHON, L.G.F., 2002. Modelo heurístico de previsão de vazões naturais médias semanais aplicado à usina de Foz do Areia. Dissertação de Mestrado. COPPE UFRJ, $88 \mathrm{p}$.

HAAN, C.T., 1989. Parametric uncertainty in hydrologic modeling. Trans. of the Am. Soc. of Agricultural Engineers, v. 32 , n. 1 , p. 137-145.

HOLLINGSWORTH, A., 2003. Improvements in the skill of numerical weather prediction with global models, Bull. World Meteorological Organization, v. 52, n. 1, p. 33-39.

IBBITT, R.P.; HENDERSON, R.D.; COPELAND, J.; WRATT, D.S., 2000. Simulating mountain runoff with mesoscale weather model rainfall estimates: a New Zealand experience. J. of Hydrol., v. 239, p. 19-32.

KOUWEN, N., SOULIS E., PIETRONIRO A., Donald J., HARRINGTON R., 1993. Grouped response units for distributed hydrologic modeling. J. of Water Res. Plan. and Manag., 119(3), 289-305.

LETTENMAIER, D.P., 2000. Macroscale Land Surface Hydrology modelling at the University of Washington. Disponivel em: http://www.ce. washington.edu/ lettenma/mlshm.html.

LIANG, X.; LETTENMAIER, D.P.; WOOD, E.F.; BURGES, S.J., 1994. A simple hydrologically based model of land surface water and energy fluxes for general circulation models. J. of Geoph. Research, v. 99, n. D7, p. $14415-14428$.

LIVINO DE CARVALHO, A.R.; COSTA, F.S.; DAMÁZIO, J.M.; GUILHON, L.G.F., 2001. Previsão de vazão diária através de uma abordagem estocástica: Modelo PREVIVAZH. XIV Simp. Bras. de Rec. Hidricos, AB$\mathrm{RH}$, Aracaju.
MACEIRA, M.E.P.; DAMÁZIO, J.M.; GHIRARDI, A.O.; DANTAS, H.M., 1997. Utilização de modelos ARMA(p,q) periódicos na previsão de vazões semanais. XII Simp. Bras. de Rec. Hídricos, ABRH, Vitória.

MAO, Q.; MUELLER, S.F.; JUANG, H-M, 2000. Quantitative precipitation forecasting for the Tennessee and Cumberland river watersheds using NCEP Regional Spectral Model. Weather and Forecasting, v. 15, p. 29-45.

McBRIDE, J.L.; EBERT, E.E., 2000. Verification of quantitative precipitation forecasts from operational numerical weather prediction models over Australia. Weather and Forecasting, v. 15, p. 103-121.

MOORE, R.J.; CLARKE, R.T., 1981. A distribution function approach to rainfall-runoff modelling. Water Res. Research, v.17, n.5.

NASH, J.; SUTCLIFFE, J., 1970. River flow forecasting through conceptual models. Part I a - Discussion of Principles. J. of Hydrol., n.1, v.10, p. 282-290.

NIJSSEM, B; LETTENMAIER, D.P.; LIANG, X.; WETZEL, S.W.; WOOD, E.F., 1997. Streamflow simulation for continental-scale river basins. Water Res. Research, v. 33 , n. 4 p. $711-724$.

PAZ, A.R.; COLLISCHONN, W.; SILVEIRA, A.L.L., 2006. Improvements in large scale drainage networks derived from digital elevation models. Water Res. Research, v.42, n.8.

PIMENTEL, A.L.G.; XAVIER, L.N.R.; COSTA, F.S.; LIMA, A.L.D.S., 2001. Usos múltiplos da água no planejamento energético da operação a curto prazo. XIV Simp. Bras. de Rec. Hídricos, ABRH, Aracaju.

REED, S.M., 2003. Deriving flow directions for coarseresolution (1-4 km) gridded hydrologic modeling. Water Res. Research, 39(9).

SHUTTLEWORTH, W.J., 1993. Evaporation. In: Maidment, D. Handbook of hydrology. C. 4, McGraw-Hill.

SOULIS E.D., KOUWEN N., PIETRONIRO A., SEGLENIEKS F.R., SNELGROVE K.R., PELLERIN P., SHAW D.W.; L. MARTZ, 2004. A Framework for Hydrological Modelling In Mags. In: Prediction in Ungauged Basins: Approaches for Canada's Cold Regions (ed. by C.Spence, J.W. Pomeroy, A.Pietroniro). CWRA ACRH Press, Ontário, Canadá.

TODINI, E., 1996. The ARNO rainfall - runoff model. J. of Hydrol., v. 175, p. 293-338.

TUCCI, C.E.M., 1998. Modelos hidrológicos. Ed. da Universidade Federal do Rio Grande do Sul - ABRH. 669 p.

TUCCI, C.E.M.; COLLISCHONN, W.; ANDREOLLI, I., 2003. Previsão de vazões de curto e médio prazo na operação de usinas hidrelétricas. Instituto de Pesquisas Hidráulicas, Universidade Federal do Rio Grande do Sul e Tractebel Energia S.A., Relatório Técnico, P\&D ANEEL.

WIGMOSTA, M.S.; VAIL, L.W.; LETTENMAIER, D.P., 1994. A distributed hydrology-vegetation model for complex 
terrain. Water Res. Research, v. 30, n. 6, p. 16651679.

WOOD, E.F.; LETTENMAIER, D.P.; ZARTARIAN, V.G., 1992. A land surface hydrology parameterization with subgrid variability for general circulation models. J. of Geoph. Research, v. 97, n. D3, p. 2717-2728.

YAPO, P.O.; GUPTA, H.V.; SOROOSHIAN, S., 1998. Multiobjective global optimization for hydrologic models. J. of Hydrol., v.204, p.83-97.

YATES, D.; WARNER, T.; LEAVESLEY, G., 2000. Prediction of a flash flood in complex terrain. Part II: A comparison of flood discharge simulations using rainfall input from radar, a dynamic model, and an automated algorithmic system. J. of App. Meteor., v.39, n.6, p. 815-825.

YU, Z.; LAKHTAKIA, M.; YARNAL, B.; WHITE, R.; MILLER, D.; FRAKES, B.; BARRON, E.; DUFFY, C.; SCHWARTZ, F., 1999. Simulating the river basin response to atmospheric forcing by linking a mesoscale meteorological model and hydrological model system. J. of Hydrol., v. 218, p. 72-91.

ZHAO, R.J., 1992. The Xinanjiang model applied in China. J. of Hydrol., v. 134, p. 371-381.

Distributed Hydrological Model for Streamflow Forecasting in the Paranaiba Basin Between Itumbiara and São Simão

\footnotetext{
ABSTRACT

This paper presents a methodology for real-time streamflow forecasting based on a distributed hydrological model, using data of observed rainfall measured at raingauges and rainfall forecast by the ETA model running at the Weather Forecasting Center of the National Institute for Space Research. State variables of the hydrological model are updated according to a scheme which uses observed streamflow data that would be available in a operational application. The methodology was tested in the Paranaiba river basin, along the reach between Itumbiara and São Simão hydroelectric power plants. Results show that the addition of quantitative rainfall forecasts may lead to a significant reduction in operational streamflow forecasting errors in this basin.

Key-words: distributed hydrological model, streamflow forecasting, rainfall forecasting.
} 